\title{
Fahasalaman'Ny Zaza Sy Ny Fampiasana Zavamaniry Mahasitrana Ao Amin'Ny Tananan'Ny Sahamamy, Mahevelona, Toamasina
}

\section{Fampidirina}

Efa ela no nisian'ny fifandraisan'ny olombelona sy ny tontolon'ny zavamaniry manodidina azy. Eran'ny izao tontolo izao dia nisy ary misy fiovany hatrany io fifandraisana io. Anisan'izany ny fifandraisan'ny fahasalaman'ny zaza sy ny zavamaniry mahasitrana. Nanaovana fanadiahadiana mikasika io fifandraisana io ny tao amin'ny tanàna atao hoe Sahamamy, ao anatin'nyfokontany Morarano, kaominin'ny/ Mahavelona, faritanyToamasina.

Nohadihadiana tamin'izany ny karazana fitsaboana avy amin'ny zavamaniry misy ao, ny halaviran'ny toerana hitsaboana, ny fahafantarana ny zavamaniry mahasitrana, ny isan'ny ankizy isan-tokan-trano, ny taonan'ny reny sy ny fotoana nonenany tao Sahamamy, ary ny fari-pahaizanyao an-tokan-trano.

Marihina fa ny fanadiahadiana dia tafiditra taoo anatin'ny atrik'asa "Ethnobotanie" izay nokarakarain'ny Missouri Botanical Garden (MBG) avy any Etazonia sy ny Karoka Nasionaly Ampiharana ny Raki-Panafody (KNARP), ary novatsian'ny National Institute of Health vola tamin'ny alalan'ny tetik'asa International Cooperative of Biodiversity Group (ICBG) izay tarihin'i Pr David Kingston.

\section{Toerana nanaovana ny fanadihadiana}

Sahamamy dia tanàna ao amin'ny Kominina ambanivohitra Mahavelona (Foulpointe) anatin'ny faritanin'i Toamasina, eo amin'ny longitida atsinanana $49^{\circ} 25^{\prime} 39.2^{\prime \prime}$ sy latitida atsimo $17^{\circ} 43^{\prime} 53.1^{\prime \prime}$, amin'ny haavo $31 \mathrm{~m}$ ambonin'ny ranomasina ary valo kilaometatra $(8 \mathrm{~km})$ andrefan'ny tanànan'ny Mahavelona..

\section{Fomba fiasa}

Fanontaniana mitovy no napetraka isan-tokan-trano hahafantarana ny momba ny fahasalaman'ny zaza sy ny fampiasana ny zavamaniry mahasitrana. Ny fotoana nanatanterahana ny asa dia nanomboka ny faha 31 martsa 2005 ka hatramin'ny faha 2 aprily 2005. Marihina fa alohan'ny niasana dia nangataka fahazoan-dalana am-bava tamin'ny Tangalamena tao an-tanàna. Rehefa nilazalaza ny momba ny fanadihadiana tamin'ny mponina ao an-tanàna dia roso tamin'ny fitetezana tokan-trano. Ireo zavamaniry mahasitrana voatonona nandritra ny fanadiahadiana dia nakana santionany tany anaty ala mba ahafantarana ny karazan'ny zavamaniry (Tabilao 1). Nisy koa ny fanadihadiana tany amin'ny toeram-pitsaboana tao Mahavelona mba hahalalana ireo aretina mpahazo ny zaza amin'iny faritra iny (Tabilao 2 ).

\section{Vokatra}

Voalaza sy voasoritra amin'ny kisary etsy ambany ny vokatra azo tamin'ny fanadihadiana. Hita soritra amin'ny Kisary 1 fa misy tokan-trano 8 mamonjy ny hopitaly na toeram-pitsaboana amin'ny fomba tandrefana rehefa misy marary. Misy tokan-trano 6 manatona mpitsabo gasy izay

\section{Correspondence}

Thierry Razafindrabeaza, KNARP, BP 702, Antanànarivo 101, MADAGASIKARA.

Pascal Rabeson, Valbio, BP 33, Ranomafana, Ifanadiana, Fianarantsoa, MADAGASIKARA.

Coco, Bruno Sabotsilahy, Zaza Torine, Jean Claude, Bany, Jaqueline, Angeline, Clément, Jeannette, Donah, Justin, Sahamamy, Fokontany Morarano, Mahavelona,Toamasina MADAGASIKARA

Ethnobotany Research \& Applications 3:353-357 (2005) 
mampiasa ny fomba nentimpaharazana raha misy marary. Vitsy ny tokan-trano manao dokotera tena (tokan-trano iray).

Ny mampiasa zavamaniry mahasitrana alaina any anaty ala betsaka kokoa noho ny mampiasa ny faritra manodidina ny tanàna (Kisary 2). Ny atsasaky ny tokan-trano nohadihadiana dia mahafantatra zavamaniry mahasitrana. Ny atsasany kosa dia na mahafantatra na tsy mahafantatra mihitsy (Kisary 3). Telo amin'ireo tokan-trano no misy ankizy mihoatra ny dimy (Kisary 4). Manodidina eo amin'ny 30 taona eo kosa ny taonan'ny reny ao an-tanàna (Kisary 5). Ny ankamaroan'ny mponina ao Sahamamy dia nonina tao antoerana mihoatra ny 10 taona (Kisary 6). Tsy nahavita afatsy fanabeazana fototra fara-fahabetsany ny renin'ny zaza ao Sahamamy (Kisary 7). Ny aretina matetika mpahazo ny olona manatona hopitaly eto Mahavelona dia ny tazo, ka ny volana Janoary sy Febroary no tena itrangany (Kisary 8, 9).

Tabilao 1. Lisitry ny zavamaniry mahasitrana fampiasa.

\begin{tabular}{|l|l|}
\hline Hampiasain'ny mponina & $\begin{array}{l}\text { Hampiasain'ny mpitsabo } \\
\text { gasy }\end{array}$ \\
\hline Amborasaha & Hazomamy \\
\hline Tsipitika & Gavoala \\
\hline Tavolo & Sahintsy \\
\hline Fotsiavadika & Tsilavondrivotra \\
\hline Tongomborina & Vahimarara \\
\hline Hazoambo & Vahimatio \\
\hline Vahivy & Sombitrorana \\
\hline Tambakombakondranodisa & \\
\hline Bonaka & \\
\hline
\end{tabular}

Tabilao 2. Aretina matetika mpahazo ny mponina eto Mahavelona.

\begin{tabular}{|l|l|c|c|c|c|}
\hline Volana & Ny aretina & \multicolumn{2}{|c|}{ Taona $\mathbf{2 0 0 3}$} & \multicolumn{2}{c|}{ Taona 2004 } \\
\cline { 3 - 6 } & & $\begin{array}{c}\mathbf{1 - 4} \\
\text { taona }\end{array}$ & $\begin{array}{c}\mathbf{> 5} \\
\text { taona }\end{array}$ & $\begin{array}{c}\mathbf{1 - 4} \\
\text { taona }\end{array}$ & $\begin{array}{c}\mathbf{> 5} \\
\text { taona }\end{array}$ \\
\hline Jan & Tazo & 128 & 164 & 75 & 62 \\
\hline Feb & Tazo & 167 & 216 & 80 & 106 \\
\hline Mar & Tazo & 69 & 69 & 64 & 60 \\
\hline Apr & Tazo & 44 & 61 & 22 & 42 \\
\hline May & Tazo & 26 & 39 & 32 & 30 \\
\hline Jon & Tazo & 17 & 49 & 14 & 23 \\
\hline Jol & Tazo & 23 & 49 & 17 & 21 \\
\hline Aog & Tazo & 20 & 52 & 28 & 31 \\
\hline Sep & Tazo & 26 & 34 & 17 & 19 \\
\hline Okt & Tazo & 22 & 50 & 24 & 32 \\
\hline Nov & Tazo & 29 & 44 & 38 & 39 \\
\hline Des & Tazo & 58 & 56 & 30 & 42 \\
\hline
\end{tabular}

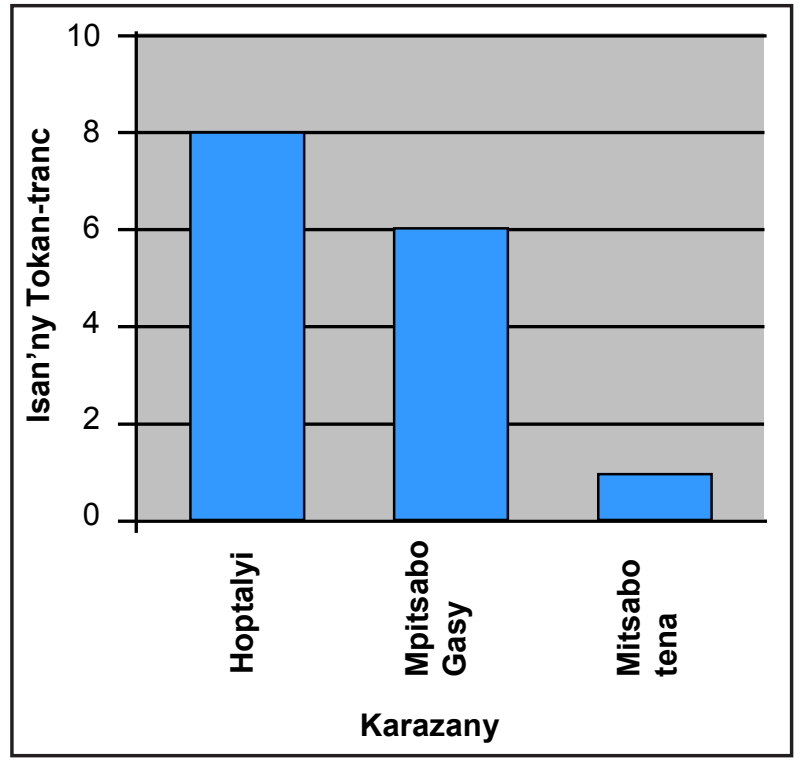

Kisary 1. Karazana fitsaboana.

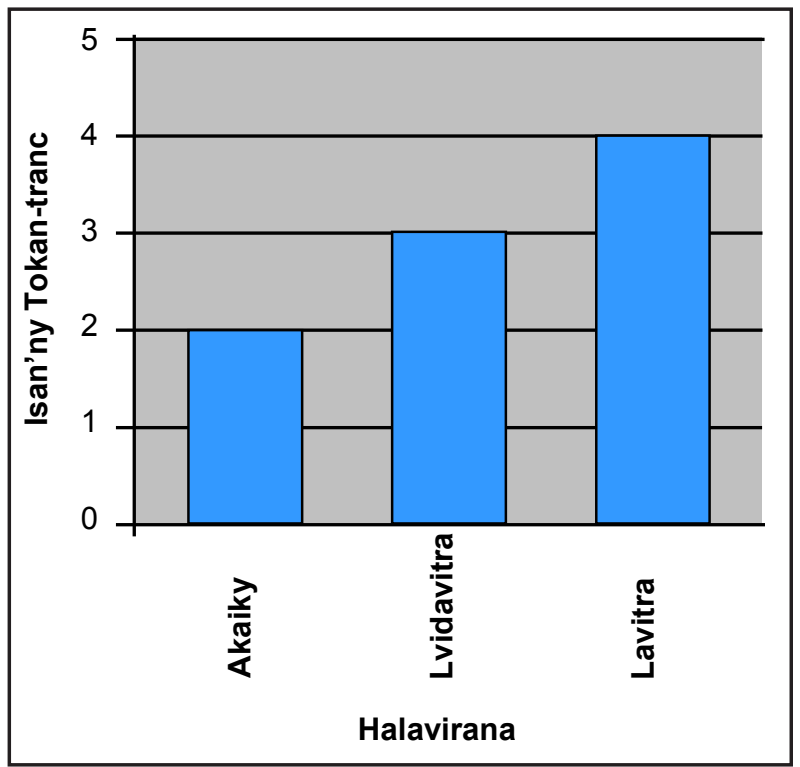

Kisary 2. Halaviran'ny toerana itsaboana.

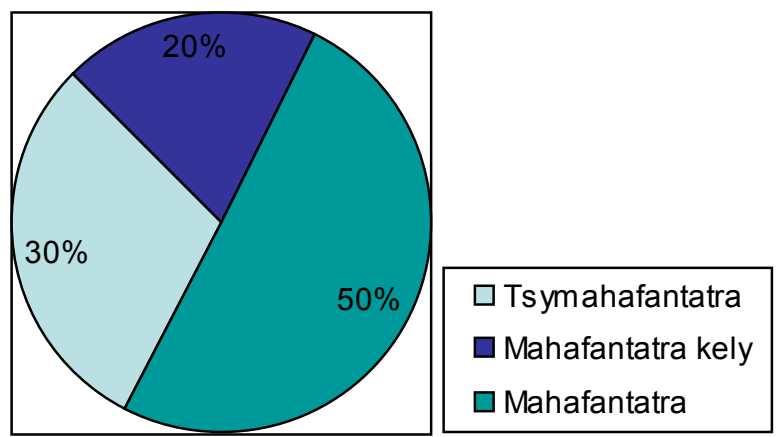

Kisary 3. Fahafantarana zavamaniry mahasitrana. 


\section{Razafindrabeaza et al. - Fahasalaman'Ny Zaza Sy Ny Fampiasana Zavamaniry \\ Mahasitrana Ao Amin'Ny Tananan'Ny Sahamamy, Mahevelona, Toamasina}

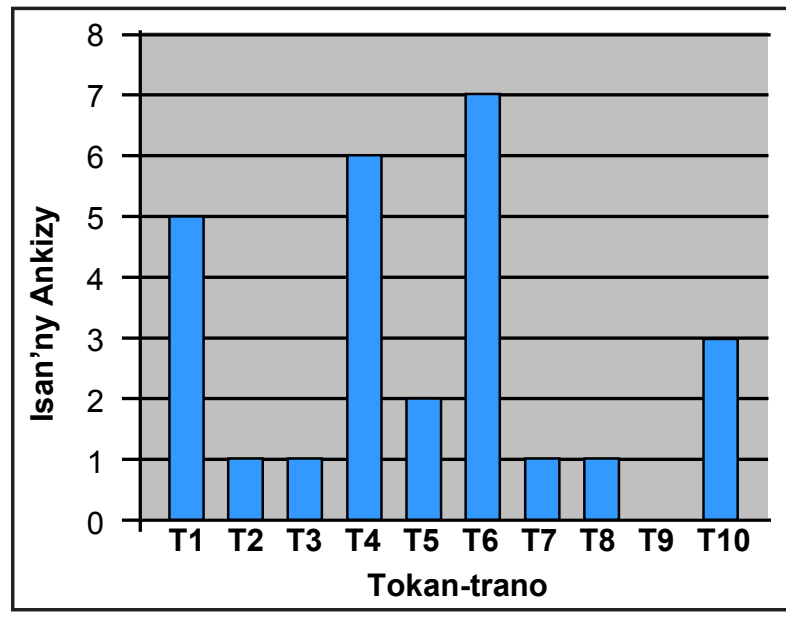

Kisary 4. Isan'ny Ankinzy ao an takan-trano.

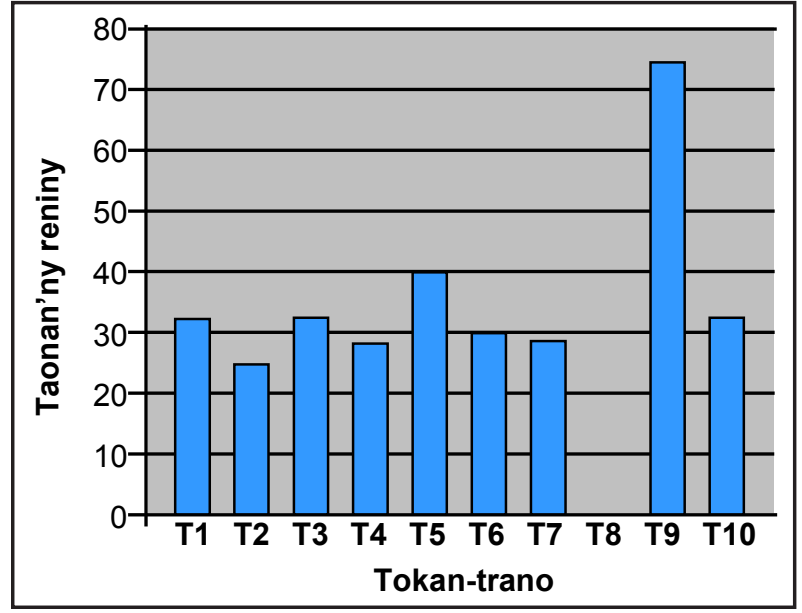

Kisary 5. Taonan'ny reniny.

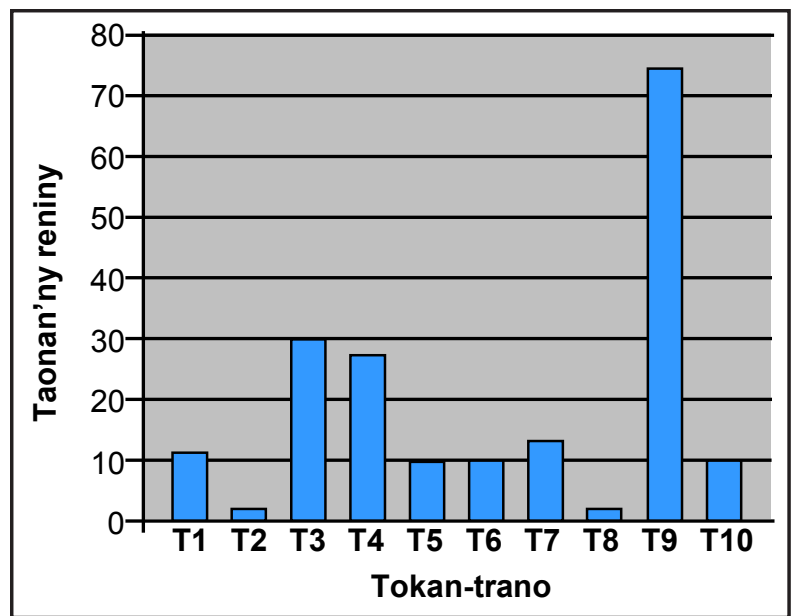

Kisary 6. Fotoana nonenany tao Sahamamy.

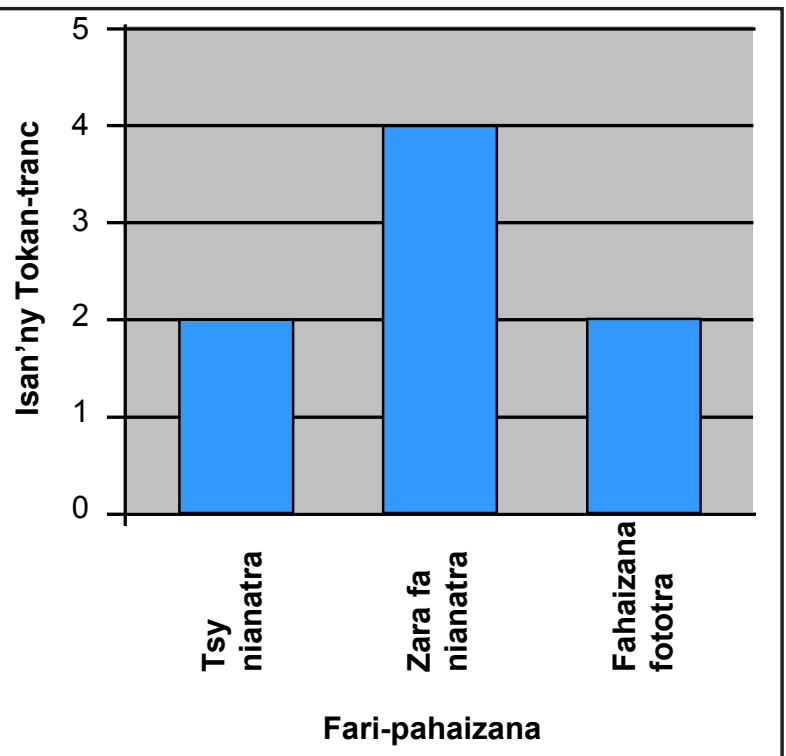

Kisary 7. Fari pahaizan'ny reniny.

Ny aretina faharoa mateti-pitranga dia ny voina (ohatra: tapaka sns...).

\section{Kirankiran-Kevitra}

Raha dinihina ny vokatra azo dia ireto manaraka ireto ny fanamarihana azo atao:

* Hita taratra fa ny ankamaroan'ny olona dia manatona hopitaly rehefa misy marary ao amin'ny fianakaviana. $\mathrm{Na}$ izany aza anefa dia be ireo manatona mpitsabo gasy rehefa mitranga ny aretina. Raha ny fahitana azy moa dia aleon'ny mponina manantona ny hopitaly amin'ny fahazoana fahasalamana. Etsy andaniny izany anefa dia lafo ho an-dry zareo ny hopitaly ka dia manatona ny mpitsabo gasy ry zareo amin'izany satria voalohany aloha dia tsy dia lavitra an-dry zareo ny fisian'ny mpitsabo ary mora kokoa ny vola aloa amin'ny fitsaboana.

* Na dia fantatra aza fa betsaka ny isan-tokan-trano no mahay karazana fanafody mahasitrana dia tsy dia manao dokotera tena loatra ny mponina ao Sahamamy fa na mandeha mankany amin'ny hopitaly, na manatona mpitsabo gasy. Eo ihany angamba ny tsy fahatokisan-dry zareo tena na koa eo ny fahafantarany fa misy hasiny ny fitsaboana ka na dia hain'ny mponina aza ny ravin-kazo mahasitrana ny hasiny kosa dia mbola mitoetra ao amin'ny mpitsabo ka dia tsy maintsy manatona ny mpitsabo ihany ry zareo rehefa mitranga ny aretina.

Raha ho an'ireo mampiasa ny zavamaniry mahasitrana moa dia fantatra fa any anaty ala no betsaka kokoa nohon'ny eo amin'ny manodidina ny tanàna. Izany angamba mampiseho ny mahasarobidy ny ala ho an'ny mponi- 


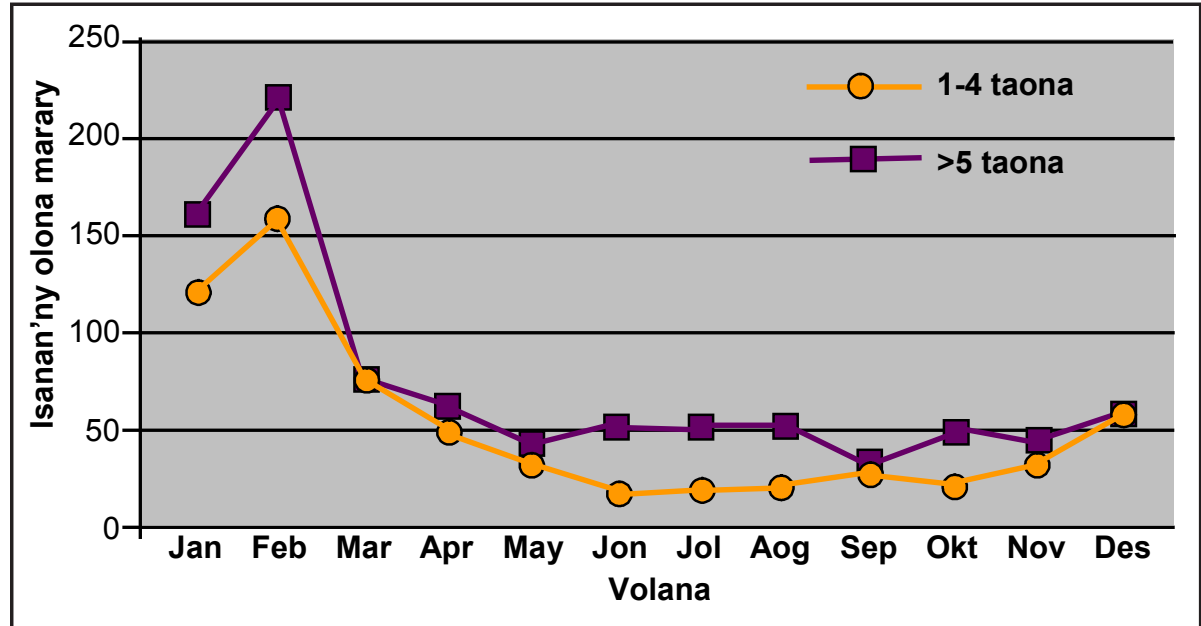

Kisary 8. Ny aretina matetika mpahazo tanoa 2003.

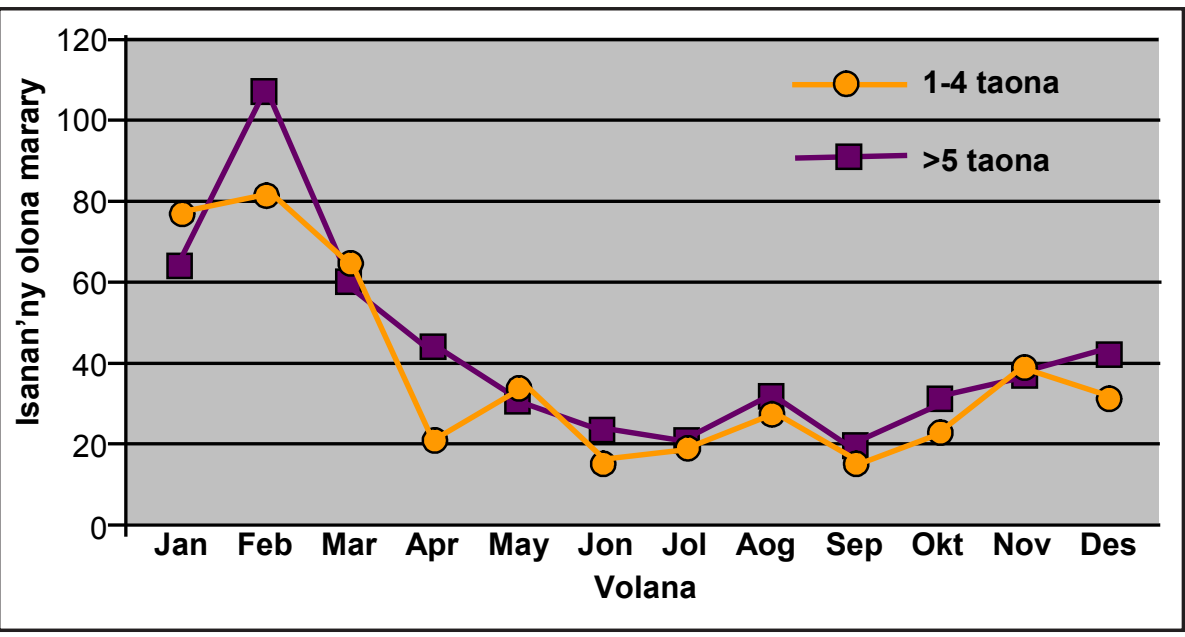

Kisary 9. Ny aretina matetika mpahazo tanoa 2004.

na mipetraka eo amin'ny manodidina. Nefa na izany aza, azo lazaina fa tsy atahorana ny hoe hakan-dry zareo betsaka any anaty ala nohon'ny fahatsapan'ny mponina fa ny mpitsabo ihany no tena mahafantatra ny fomba fampiasana ireo zavamaniry mahasitrana ka tsy ny mponina rehetra akory no mandeha any anaty ala maka azy..

*Tsy azo lazaina fa vitsy ny tokan-trano manana zaza mihoatra ny dimy ny isany ao an-tokan-trano. Ny antony moa tsy dia mazava loatra na mety misy fiantraikany amin'ny fanabeazana aizana na tsia. Ny fotoana koa moa tsy ampy nanadiahadiana raha toa ka misy izany fampianarana izany. Nisy ihany anefa no nilaza fa misy ireo dokotera izay mandalo tsindraindray mitety tanàna ka asa raha ireo no manampy amin'ny fampianarana an-dry zareo.

Raha ny fari-pahaizan'ny ankamaroan'ny reny ao antanàna dia fanabeazana fototra no tena azon'ny ankamaroany. Angamba tsy ho ampy izany fahalalana izany mba hahafahan-dry zareo mamantatra ny fomba fanabeazana aizana mba tsy hiteraka betsaka. Ny zavatra hita anefa dia tsy betsaka ny t okan-trano be ankizy ao an-tanana. Ny zavatra azo marihina ihany koa dia taranaka olona mpianadahy vitsivitsy ny ankamaroan-dry zareo ao antanàna. Raha izany moa no zohina dia avy any ivelan'ny tanàna no betsaka alain'ny tanora vady ka mety misy ny fahafantaran-dry zareo avy any amin'ny tany nihaviany ny tsy fiterahana betsaka. Vinavina ihany izany fa ny fanadihadiana moa dia voafetra tamin'ny fotoana ary ny tanàna ihany koa dia kely ka sarotra ny manamarina an'izany vinavina izany.

Etsy andaniny dia ny tazo no tena aretina mpahazo ny mponina ao Sahamamy. Araka ny fantatra dia faritra mando sy mafana ny amin'iny faritra atsinanana iny ka izay angamba no tena mahabe ny tazo ao an-tanàna ao. Voamarin'ny tao amin'ny hopitaly fa tazo no tena betsaka ao amin'ny faritr'i Foulpointe ary amin'ny fotoana mahabe 


\section{Razafindrabeaza et al. - Fahasalaman'Ny Zaza Sy Ny Fampiasana Zavamaniry Mahasitrana Ao Amin'Ny Tananan'Ny Sahamamy, Mahevelona, Toamasina}

ny orana ny mafana mihintsy no tena itrangany (volana Janoary sy Febroary).

\section{Famintinana}

Iny fanadiahadiana tao anatin'ny fotoana fohy iny dia nahitana fa na teo aza ny fahafantaran'ny mponina ao antanàna ny zavamaniry mahasitrana dia mbola ny hopitaly no vonjen'ny olona voalohany raha vantany misy marary. Noho ny fahalafosan'ny vidin'ny fitsaboana any amin'ny hopitaly anefa dia manatona mpitsabo gasy ry zareo satria moramora kokoa ny fizahana sy ny fitsaboana.

Tsy dia sahy loatra manao dokotera tena anefa ny olona ao Sahamamy dia hainy aza ny karazan'ireo zavamaniry mahasitrana satria mbola eo dia eo ny fanajany an'ireo mpitsabo gasy nohon'ny hasina misy ao aminy hahafahany mitsabo.

Tokony hifantoka amin'ny fiarovana sy ny fanafoanana ny tazomoka ny fanampiana atao amin'ny mponina ao Sahamamy sy ny manodidina ary tokony mba halefahana ihany ny saran'ny fitsaboana any amin'ny hopitaly satria tsy takatry ny mponina izany ka sahirana ry zareo rehefa mitranga ny aretina.
Eto am-pamaranana dia tsapa fa tena ilaina mihintsy ny fanadihadiana toy izao hahafahana mamantatra ny zavamisy marina iainan'ny olona any ambanivolo rehetra any. Ilaina ihany koa hahafantarana ny hevitry ry zareo ao an-toerana mba hampahomby ny fanapahan-kevitra raisin'ny aty amin'ny fanjakana indrindra indrindra raha ifandraisan'ny mponina sy ny fiarovana ny tontolo ianana no resahina.

\section{Fisaorana}

Isaorana ny MBG sy ny KNARP nikarakara ity atrik'asa fiofanana mikasika ny taranja ethnobotanique ity. Misaotra ihany koa ny mpamatsy vola ny atrik'asa dia ny National Institute of Health sy ny International Cooperative of Biodiversity Group. Atolotra ho an'ny mpampianatra roa ny fisaorana nohon'ny fahafoizan'izy ireo fotoana sy herim-po nanatanterahana ny atrik'asa fiofanana. Fisaorana lehibe ho an'ny mponina monina tao Sahamamy tamin'ny fiaraha-miasa nahafatra-po nasehon-dry zareo. Tsy adino koa ny tao amin'ny Hotel "Le vent du Sud" tao Foulpointe nohon'ny Fikarakarana tamin'ny fiantranona sy sakafo nandritra ny nipetrahana tao. Mankasitra ary misaotra manokana an'ireo mpandray anjara rehetra tamin'ity atrik'asa fiofanana ity.

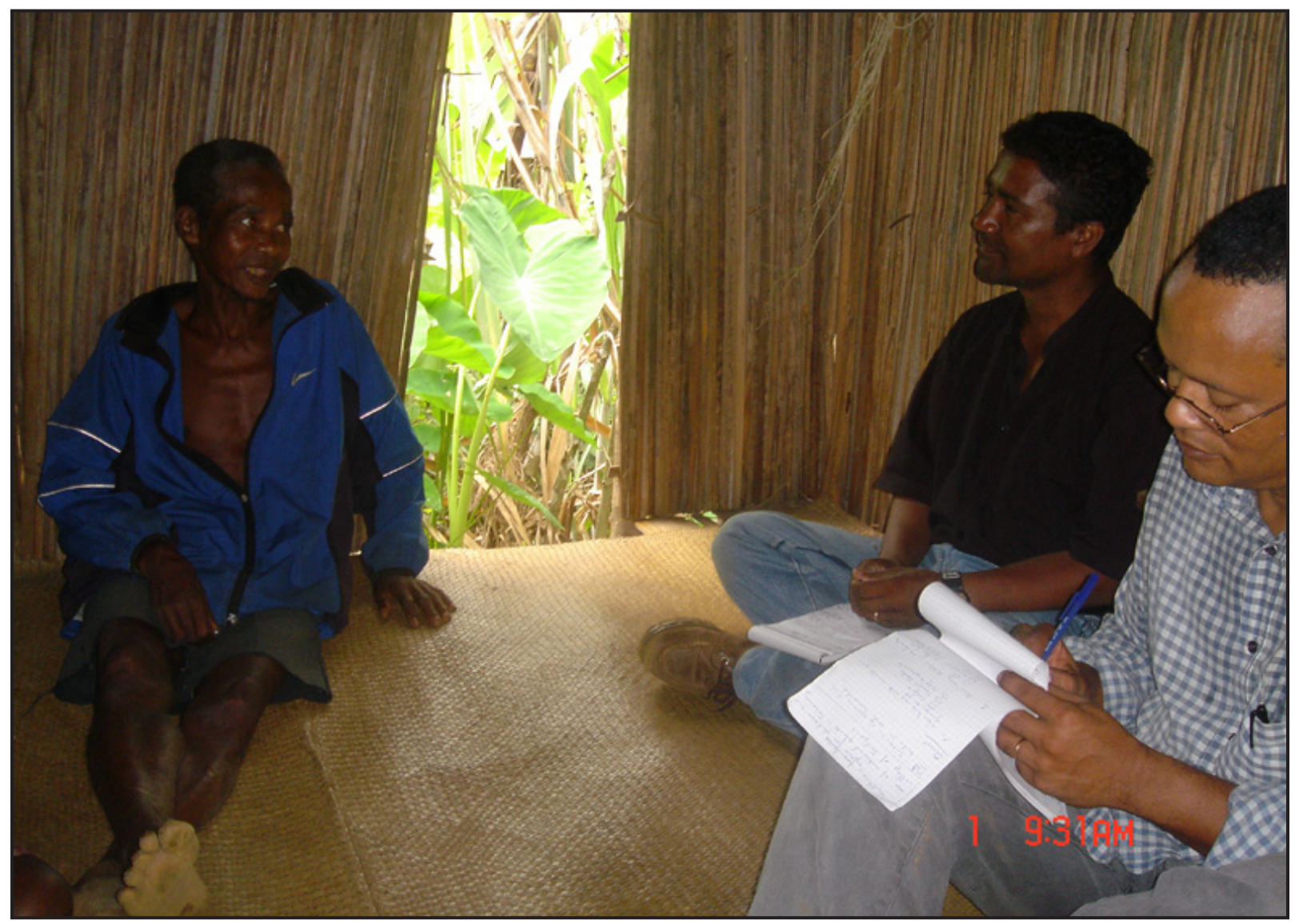


